\title{
The Need for Biological Outcomes for Biological Drugs in Psoriatic Arthritis
}

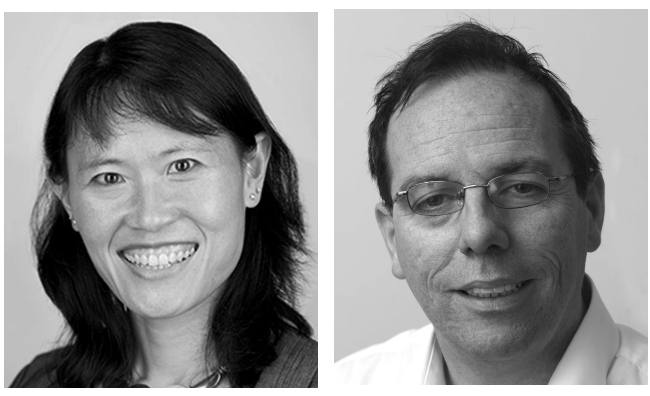

Perhaps no disease has been bedeviled more than psoriatic arthritis (PsA), with its proliferation of different clinical outcome measures. The development of these various measures to a large degree reflects both an inability to fully grasp disease pathogenic mechanisms and the difficulty in measuring them, especially in the context of the disease's clinical heterogeneity. The absence of biological outcome measures, including an immunological or inflammation-related serum marker, makes the tomographic ability of magnetic resonance imaging (MRI) to directly measure inflammation arguably the most useful surrogate for PsA biological disease assessment. This is important because outcome measures to assess PsA are mushrooming (Figure $1)$.

In this issue of The Journal, Bird and colleagues apply MRI to therapy response assessments and show that high-field MRI is more sensitive than low-field MRI for detection of MRI synovitis, tenosynovitis, and osteitis reactions ${ }^{1}$. Although small, the study should kindle interest in research efforts to accurately evaluate the role of conventional synthetic disease-modifying antirheumatic drugs (DMARD), targeted synthetic DMARD, and biological agent optimization in PsA.

Although clinically heterogeneous, PsA is characterized by inflammation at the entheses ${ }^{2}$, with secondary synovitis and osteitis consequent to enthesitis ${ }^{3,4}$. The extensive inflammatory reaction in disease is likely due to the nature of the synovio-entheseal complex, where structures adjacent to the entheses - which form part of the enthesis organ - are affected $^{5}$. Several studies have shown a high burden of enthesopathy in psoriasis, with a small study also suggesting these entheseal changes predate clinical arthritis ${ }^{6,7}$.

From experimental model systems including tumor necrosis factor (TNF) and interleukin 23 overexpression systems, it is clear that polyarticular "rheumatoid-like" disease commences at the enthesis, but with the passage of time, severe synovial-based joint destruction akin to rheumatoid arthritis (RA) is evident ${ }^{8,9}$. Recognition that the initial "blast zone" at the enthesis as its epicenter leads to diffuse soft tissue inflammation including subcutaneous inflammation, tenosynovitis, synovitis, and diffuse osteitis should help investigators define strategies to better measure the disease process.

This is all the more relevant because joint involvement in PsA can mimic other common forms of arthritis, making it difficult to differentiate PsA from degenerative arthritis $^{3}$; compared to RA, joint disease in PsA is associated with a lesser degree of pain and stiffness. The need to accurately measure all these enthesis-related articular joint-associated inflammatory manifestations is key to accurately defining true biological responses to therapy and for determining which therapies are most effective.

Given the lack of a good serum biomarker for PsA and the diffuse nature of entheseal-associated disease, it appears that imaging will play a key role in determining the true biology of the underlying disease. This is especially the case because the ever-increasing number of disease measures have clearly failed to fully define the inflammatory features of PsA (Figure 1).

In that regard, given its ability to measure all disease manifestations, including osteitis, MRI is very useful for assessment, in contrast to ultrasound. Bird, et al show how MRI can be used to evaluate early response to anti-TNF therapy in a small cohort of patients with $\mathrm{PsA}^{1}$. Comparatively inexpensive, low-field MRI was compared to the more expensive high-field MRI - because the former would be a handy surrogate for disease assessment in a real-world setting and not just confined to clinical trials. Bird, et al show good intraobserver reliability for both high- and low-field MRI. However, low-field MRI failed to demonstrate osteitis, reflecting the inferior fat suppression of the technique and poorer spatial resolution.

Their data are interesting in that they demonstrated MRI response to anti-TNF as early as within 2 weeks. This is particularly observed in the improvement in tenosynovitis, where as in previous reports, tenosynovitis tends to respond quicker than joint synovitis ${ }^{10,11}$. Flexor tenosynovitis is common in PsA, has been noted to be a prominent feature

See MRI responsiveness in PsA, page 75

Personal non-commercial use only. The Journal of Rheumatology Copyright @ 2016 . All rights reserved. 


\section{A. PsA outcome measures}

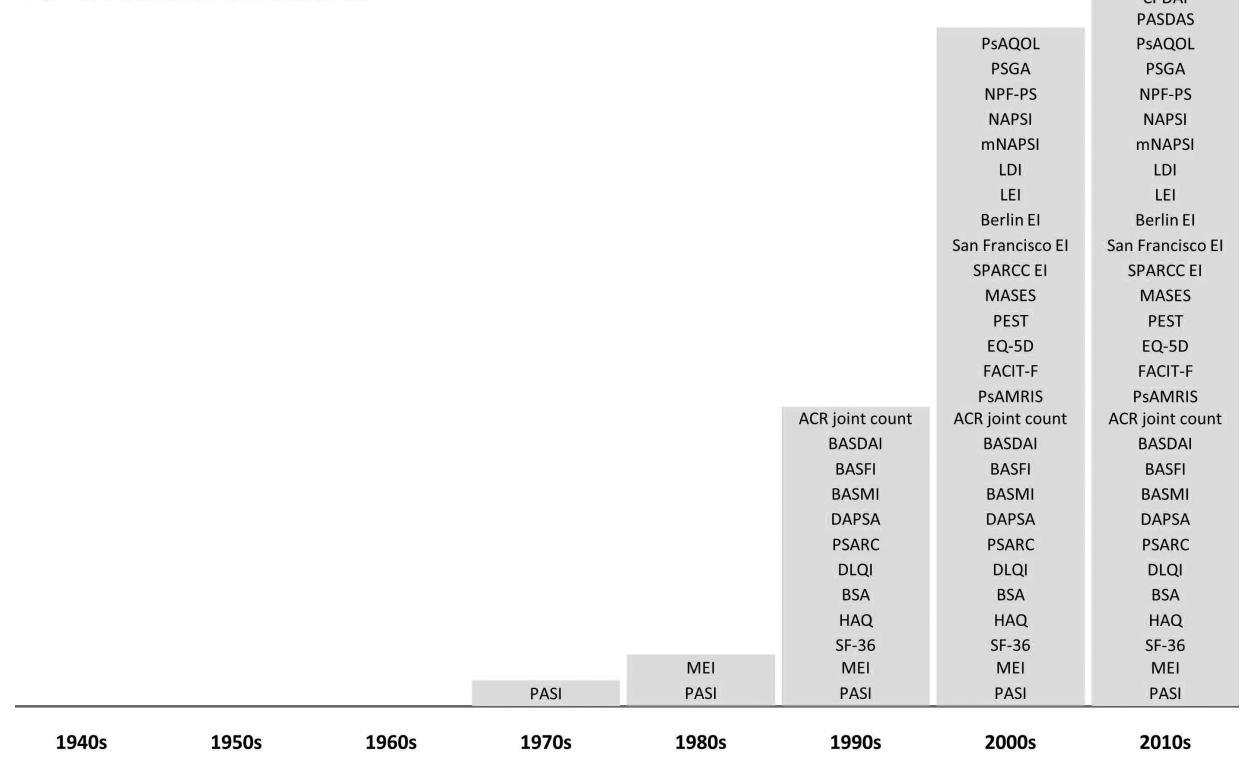

\section{B. RA outcome measures}

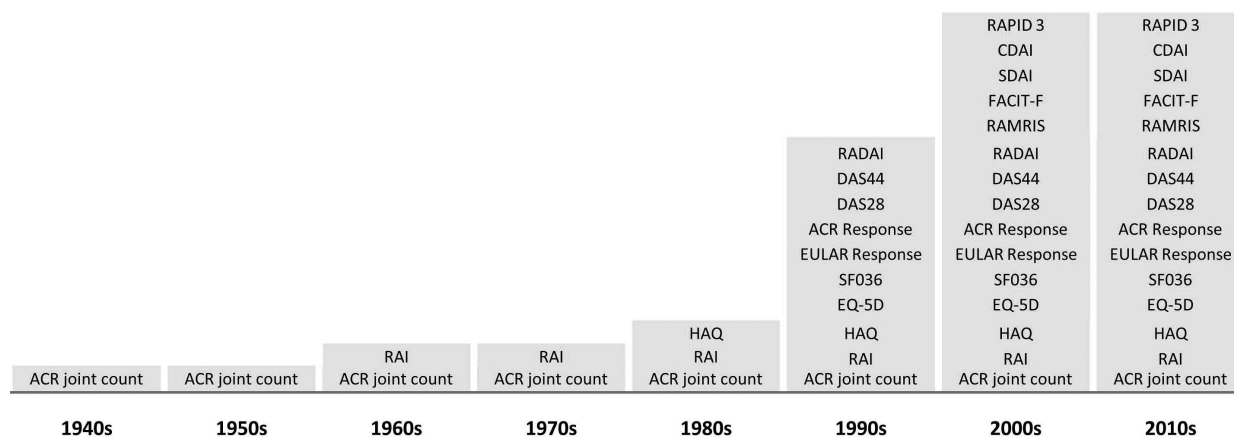

Figure 1. The use of clinical outcome measures in (A) psoriatic arthritis (PsA) compared to (B) rheumatoid arthritis (RA) over the decades ${ }^{28,30}$. There is an exponential increase in PsA outcome measures compared to RA because of the multidimensional nature of the disease, which makes it more difficult to assess adequately. The relatively more complex nature of PsA provides a challenge in developing a good tool that can measure joints, skin, nails, and entheses, resulting in the creation of multiple tools in the search for the ideal all-in-one outcome measure. Because biological drugs specifically target the immune system, MRI, with its ability to measure inflammation in different tissues, is the technique best able to measure most of the changes in PsA. The lists of outcome measures may not be exhaustive. MRI: magnetic resonance imaging; PASI: Psoriasis Area and Severity Index; MEI: Mander Enthesitis Index; SF-36: Medical Outcomes Study Short Form-36; HAQ: Health Assessment Questionnaire; BSA: body surface area; DLQI: Dermatology Life Quality Index; PsARC: Psoriatic Arthritis Response Criteria; DAPSA: Disease Activity in PsA; BASMI: Bath Ankylosing Spondylitis Metrology Index; BASFI: Bath Ankylosing Spondylitis Function Index; BASDAI: Bath Ankylosing Spondylitis Disease Activity Index; ACR: American College of Rheumatology; PsAMRIS: PsA MRI Scoring; FACIT-F: Functional Assessment of Chronic Illness Therapy-Fatigue; PEST: Psoriasis Epidemiology Screening Tool; MASES: Maastricht Ankylosing Spondylitis Enthesis Score; SPARCC: Spondyloarthritis Research Consortium of Canada; EI: Enthesitis Index; LEI: Leeds Enthesitis Index; LDI: Leeds Dactylitis Index; NAPSI: Nail Psoriasis Severity Index; mNAPSI: Modified Nail Psoriasis Severity Index; NPF-PS: National Psoriasis Foundation Psoriasis Score; PSGA: Physician Static Global Assessment; PsAQOL: PsA Quality of Life; PASDAS: PsA Disease Activity Score; CPDAI: Composite Psoriatic Disease Activity Index; PSAJAI: PsA Joint Activity Index; RAI: Ritchie Articular Index; EULAR: European League Against Rheumatism; DAS28/44: Disease Activity Score 28/44 joints; RADAI: RA Disease Activity Index; RAMRIS: RA MRI Scoring; SDAI: Simplified Disease Activity Index; CDAI: Clinical Disease Activity Index; RAPID-3: Routine Assessment of Patient Index Data 3. 
in dactylitis, and is directly linked to microenthesitis ${ }^{12,13,14}$. When compared with RA, tenosynovitis is seen more frequently in PsA, and where flexor tenosynovitis is more common than the extensor counterpart ${ }^{15,16,17}$. Nevertheless, studies in RA have also found tenosynovitis to be a feature that also responds rapidly to anti-TNF therapy ${ }^{18}$, so much so that consideration to include tenosynovitis into the Rheumatoid Arthritis Magnetic Resonance Imaging Scoring System scores for RA is on the recent OMERACT (Outcome Measures for Rheumatology initiative) research agenda ${ }^{19}$.

However, even though both PsA and RA share common general good inflammatory response to anti-TNF, the process is driven by different molecular mediators ${ }^{20,21}$. The differential vascularity in the inflammatory process in the 2 arthritides may also explain the differential responses. There is also anatomic variation to the response to therapy between RA and spondyloarthritis, including PsA, as seen in the different regions of the knee joint ${ }^{22}$. Macroscopic data have shown distinct vascular pattern in PsA compared to RA, where the vasculature was described as tortuous and bushy in $\operatorname{PsA}^{23}$. Certainly there have been reports on the histology of enthesitis in dactylitis demonstrating hypervascular tenosynovium $^{24}$. Studies have noted that dactylitis in PsA appears to respond better to some anti-TNF agents compared to others, but this may be because the outcome measures used could not measure the primary pathology of PsA - i.e., enthesitis - in a feasible and accurate manner, resulting in difficulty quantifying true biological therapy response ${ }^{25}$.

In general, the inflammatory process in PsA can be diffuse and affects multiple structures, including the extracapsular tissues, which can be more difficult to quantify, in comparison to more discrete and focal lesions such as synovitis. PsA clinical trials occasionally tend to adopt RA measures to measure outcome because more specific disease tools in PsA are lacking. This can lead to inaccurate reflections of therapy outcomes, in particular when changes such as enthesitis and extracapsular inflammation more reflective of PsA pathology are not being identified appropriately. Bird, et al used a modified Psoriatic Arthritis Magnetic Resonance Imaging Scoring System (PsAMRIS) scoring method ${ }^{26}$, and confined the analysis to changes within anatomical boundaries such as the tendons (tenosynovitis), joint capsule or synovium (synovitis), and bone (bone edema), and did not look at periarticular inflammation - a characteristic change in PsA.

Dynamic contrast-enhanced MRI (DCE-MRI), which allows quantification of synovial inflammation, is often used in RA to identify the quantity and intensity of synovitis and for therapy monitoring ${ }^{27}$. Although DCE-MRI has also been used to monitor disease in $\mathrm{PsA}^{11}$, the technique is not completely ideal for PsA, because it relies on a semiquantitative method involving a manual creation of regions of interest (ROI). Articular synovitis can be delineated relatively easily, but enthesitis is often diffuse and outside the joint capsule, and it can be difficult to visualize an outline for an
ROI. As a result, most if not all studies tend to "ignore" the soft tissue inflammation in PsA, and at best, the measurement of synovitis, tenosynovitis, bone edema, and erosions on MRI represents a "surrogate" marker for disease in PsA.

In PsA, there appear to be numerous outcome measures developed over time, suggesting that the heterogeneous nature of the disease makes creation of a single comprehensive outcome measure (that is also feasible to use) difficult (Figure 1) ${ }^{28}$. Unlike RA, a common presentation of PsA is not confined to the joint, but also the skin, nails, and entheses. In a recent systematic literature review, the Group for Research and Assessment of Psoriasis and Psoriatic Arthritis Enthesitis Working Group found that, for the assessment of enthesitis in various clinical trials in PsA, there were different enthesitis indices used in different studies ${ }^{29}$.

In the absence of a globally agreed-upon tool, Bird, et al have used the best available imaging tool for measuring disease activity in PsA - one that mirrors "true" disease activity as closely as possible - for now. High-field MRI appears to be better if osteitis is to be evaluated (which should always be assessed because it may be a forerunner of joint erosion). Although the modified PsAMRIS scores used were adequate to show a change with therapy, it will be useful to see more trials adopting the full PsAMRIS scoring, which includes periarticular changes that are more representative of PsA.
AI LYN TAN, MD, MRCP,
Associate Professor and Consultant in Rheumatology;
DENNIS McGONAGLE, PhD, FRCPI,
Professor in Regenerative Medicine,
UK National Institute for Health Research
Leeds Musculoskeletal Biomedical Research Unit,
Chapel Allerton Hospital,
Leeds Institute of Rheumatic and Musculoskeletal Medicine, University of Leeds,
Leeds, UK.

Address correspondence to Dr. A.L. Tan, Leeds Institute of Rheumatic and Musculoskeletal Medicine, Chapel Allerton Hospital, Chapeltown Road, Leeds LS7 4SA, UK; E-mail: a.1.tan@leeds.ac.uk

\section{REFERENCES}

1. Feletar M, Hall S, Bird P. Evaluation of magnetic resonance imaging responsiveness in active psoriatic arthritis at multiple timepoints during the first 12 weeks of antitumor necrosis factor therapy. J Rheumatol 2016;43:75-80.

2. McGonagle D, Gibbon W, Emery P. Classification of inflammatory arthritis by enthesitis. Lancet 1998;352:1137-40.

3. Tan AL, Grainger AJ, Tanner SF, Emery P, McGonagle D. A high-resolution magnetic resonance imaging study of distal interphalangeal joint arthropathy in psoriatic arthritis and osteoarthritis: are they the same? Arthritis Rheum 2006;54:1328-33.

4. Lories RJ, Matthys P, de Vlam K, Derese I, Luyten FP. Ankylosing enthesitis, dactylitis, and onychoperiostitis in male DBA/1 mice: a model of psoriatic arthritis. Ann Rheum Dis 2004;63:595-8.

5. McGonagle D, Lories RJ, Tan AL, Benjamin M. The concept of a "synovio-entheseal complex" and its implications for understanding

Personal non-commercial use only. The Journal of Rheumatology Copyright (C) 2016. All rights reserved. 
joint inflammation and damage in psoriatic arthritis and beyond. Arthritis Rheum 2007;56:2482-91.

6. Gisondi P, Tinazzi I, El-Dalati G, Gallo M, Biasi D, Barbara LM, et al. Lower limb enthesopathy in patients with psoriasis without clinical signs of arthropathy: a hospital-based case-control study. Ann Rheum Dis 2008;67:26-30.

7. Ash ZR, Tinazzi I, Gallego CC, Kwok C, Wilson C, Goodfield M, et al. Psoriasis patients with nail disease have a greater magnitude of underlying systemic subclinical enthesopathy than those with normal nails. Ann Rheum Dis 2012;71:553-6.

8. Lubberts E. The IL-23-IL-17 axis in inflammatory arthritis. Nat Rev Rheumatol 2015;11:415-29.

9. Bluml S, Scheinecker C, Smolen JS, Redlich K. Targeting TNF receptors in rheumatoid arthritis. Int Immunol 2012;24:275-81.

10. Yanaba K, Sadaoka A, Yonenaga T, Saeki H, Umezawa Y, Tojo S, et al. Adalimumab markedly improves enthesitis in patients with psoriatic arthritis: evaluation with a magnetic resonance imaging scoring system. J Dermatol 2015 Jun 24 (E-pub ahead of print).

11. Poggenborg RP, Wiell C, Boyesen P, Boonen A, Bird P, Pedersen SJ, et al. No overall damage progression despite persistent inflammation in adalimumab-treated psoriatic arthritis patients: results from an investigator-initiated 48 -week comparative magnetic resonance imaging, computed tomography and radiography trial. Rheumatology 2014;53:746-56.

12. Bakewell CJ, Olivieri I, Aydin SZ, Dejaco C, Ikeda K, Gutierrez M, et al. Ultrasound and magnetic resonance imaging in the evaluation of psoriatic dactylitis: status and perspectives. J Rheumatol 2013;40:1951-7.

13. Healy PJ, Groves C, Chandramohan M, Helliwell PS. MRI changes in psoriatic dactylitis - extent of pathology, relationship to tenderness and correlation with clinical indices. Rheumatology 2008;47:92-5.

14. Tan AL, Fukuba E, Halliday NA, Tanner SF, Emery P, McGonagle D. High-resolution MRI assessment of dactylitis in psoriatic arthritis shows flexor tendon pulley and sheath-related enthesitis. Ann Rheum Dis 2015;74:185-9.

15. Lin Z, Wang Y, Mei Y, Zhao Y, Zhang Z. High-frequency ultrasound in the evaluation of psoriatic arthritis: a clinical study. Am J Med Sci 2015;350:42-6.

16. Narvaez J, Narvaez JA, de Albert M, Gomez-Vaquero C, Nolla JM. Can magnetic resonance imaging of the hand and wrist differentiate between rheumatoid arthritis and psoriatic arthritis in the early stages of the disease? Semin Arthritis Rheum 2012;42:234-45.

17. Yonenaga T, Saeki H, Nakagawa H, Fukuchi O, Umezawa Y, Hayashi M, et al. Four cases of Japanese patients with psoriatic arthritis in whom effective treatments by anti-tumor necrosis factor-alpha drugs were evaluated by magnetic resonance imaging together with improvement of skin lesions. J Dermatol 2015; 42:49-55

18. Axelsen MB, Eshed I, Horslev-Petersen K, Stengaard-Pedersen K, Hetland ML, Moller J, et al. A treat-to-target strategy with methotrexate and intra-articular triamcinolone with or without adalimumab effectively reduces MRI synovitis, osteitis and tenosynovitis and halts structural damage progression in early rheumatoid arthritis: results from the OPERA randomised controlled trial. Ann Rheum Dis 2015;74:867-75.

19. Ostergaard M, Bird P, Gandjbakhch F, Eshed I, Haugen IK, Haavardsholm EA, et al. The OMERACT MRI in Arthritis Working Group - update on status and future research priorities. J Rheumatol 2015 Feb 15 (E-pub ahead of print).

20. Lories RJ, Baeten DL. Differences in pathophysiology between rheumatoid arthritis and ankylosing spondylitis. Clin Exp Rheumatol 2009;27 Suppl 55:S10-4.

21. Dougados M, Baeten D. Spondyloarthritis. Lancet 2011; 377:2127-37.

22. Rhodes LA, Tan AL, Tanner SF, Radjenovic A, Hensor EM, Reece $\mathrm{R}$, et al. Regional variation and differential response to therapy for knee synovitis adjacent to the cartilage-pannus junction and suprapatellar pouch in inflammatory arthritis: implications for pathogenesis and treatment. Arthritis Rheum 2004;50:2428-32.

23. Reece RJ, Canete JD, Parsons WJ, Emery P, Veale DJ. Distinct vascular patterns of early synovitis in psoriatic, reactive, and rheumatoid arthritis. Arthritis Rheum 1999;42:1481-4.

24. Tuttle KS, Vargas SO, Callahan MJ, Bae DS, Nigrovic PA. Enthesitis as a component of dactylitis in psoriatic juvenile idiopathic arthritis: histology of an established clinical entity. Pediatr Rheumatol Online J 2015;13:7.

25. Rose S, Toloza S, Bautista-Molano W, Helliwell PS. Comprehensive treatment of dactylitis in psoriatic arthritis. J Rheumatol 2014;41:2295-300.

26. Ostergaard M, McQueen F, Wiell C, Bird P, Boyesen P, Ejbjerg B, et al. The OMERACT psoriatic arthritis magnetic resonance imaging scoring system (PsAMRIS): definitions of key pathologies, suggested MRI sequences, and preliminary scoring system for PsA Hands. J Rheumatol 2009;36:1816-24.

27. Borrero CG, Mountz JM, Mountz JD. Emerging MRI methods in rheumatoid arthritis. Nat Rev Rheumatol 2011;7:85-95.

28. Mease PJ. Measures of psoriatic arthritis: Tender and Swollen Joint Assessment, Psoriasis Area and Severity Index (PASI), Nail Psoriasis Severity Index (NAPSI), Modified Nail Psoriasis Severity Index (mNAPSI), Mander/Newcastle Enthesitis Index (MEI), Leeds Enthesitis Index (LEI), Spondyloarthritis Research Consortium of Canada (SPARCC), Maastricht Ankylosing Spondylitis Enthesis Score (MASES), Leeds Dactylitis Index (LDI), Patient Global for Psoriatic Arthritis, Dermatology Life Quality Index (DLQI), Psoriatic Arthritis Quality of Life (PsAQOL), Functional Assessment of Chronic Illness Therapy-Fatigue (FACIT-F), Psoriatic Arthritis Response Criteria (PsARC), Psoriatic Arthritis Joint Activity Index (PsAJAI), Disease Activity in Psoriatic Arthritis (DAPSA), and Composite Psoriatic Disease Activity Index (CPDAI). Arthritis Care Res 2011;63 Suppl 11:S64-85.

29. Orbai AM, Weitz J, Siegel EL, Siebert S, Savage LJ, Aydin SZ, et al. Systematic review of treatment effectiveness and outcome measures for enthesitis in psoriatic arthritis. J Rheumatol 2014;41:2290-4.

30. Hobbs KF, Cohen MD. Rheumatoid arthritis disease measurement: a new old idea. Rheumatology 2012;51 Suppl 6:vi21-7.

J Rheumatol 2016;43:3-6; doi:10.3899/jrheum.151296 